University of Wollongong

Research Online

Faculty of Engineering - Papers (Archive)

Faculty of Engineering and Information

Sciences

2003

\title{
Readout of LYSO using a new silicon photodetector for positron emission tomography
}

M. L. Lerch

University of Wollongong, mlerch@uow.edu.au

R. Ward

University of Wollongong

Philip E. Simmonds

University of Wollongong, simmonds@uow.edu.au

V. L. Perevertailo

SPA-BIT "Detector", Kiev, Ukraine

S. R. Meikle

Royal Prince Alfred Hospital, Sydney

See next page for additional authors

Follow this and additional works at: https://ro.uow.edu.au/engpapers

Part of the Engineering Commons

https://ro.uow.edu.au/engpapers/49

\section{Recommended Citation}

Lerch, M. L.; Ward, R.; Simmonds, Philip E.; Perevertailo, V. L.; Meikle, S. R.; Taylor, G. N.; and Rosenfeld, Anatoly B.: Readout of LYSO using a new silicon photodetector for positron emission tomography 2003. https://ro.uow.edu.au/engpapers/49 
Authors

M. L. Lerch, R. Ward, Philip E. Simmonds, V. L. Perevertailo, S. R. Meikle, G. N. Taylor, and Anatoly B. Rosenfeld 


\title{
Readout of LYSO Using a New Silicon Photodetector for Positron Emission Tomography
}

\author{
Michael L.F. Lerch, Member, IEEE, Robert Ward Student Member, IEEE, Phillip E. Simmonds, \\ Vladimir L. Pervertailo, Steven R. Meikle, Geoffery N. Taylor and Anatoly B. Rosenfeld, Senior \\ Member, IEEE
}

\begin{abstract}
This article focuses on the characterisation of three versions of a custom designed silicon photodetector using nuclear spectroscopy techniques. Room temperature gamma ray spectroscopy experiments have been performed using an LYSO excited by ${ }^{137} \mathrm{Cs}$ and ${ }^{22} \mathrm{Na}$ sources and read out by the new photodiodes. We have measured an energy resolution of $13.1 \%$ FWHM for the $511 \mathrm{keV}$ gamma rays from a ${ }^{22} \mathrm{Na}$ source and $11.2 \%$ FWHM for the $662 \mathrm{keV}$ gamma rays from a ${ }^{137} \mathrm{Cs}$ source. This is one of the best ever-reported energy resolutions for a lutetium based scintillator crystal read out by a silicon photodetector.
\end{abstract}

\section{INTRODUCTION}

Current commercial PET scanners are general purpose devices which are designed to support a wide range of clinical applications. These generally require a scanner with reasonably large detector ring diameter $(\geq 80 \mathrm{~cm})$ and the ability to image the whole body in incremental steps. Recently, new applications of PET have emerged which place different demands on PET instrumentation, requiring high spatial resolution and sensitivity, in particular for imaging small volumes. Such applications include small animal imaging, breast cancer imaging and high resolution brain imaging. With these emerging applications, a large ring diameter is not only not required but also limits the performance that can be achieved when imaging small volumes.

Manuscript received October 7, 2003. This work was supported in part by the Australian National Health and Medical Research Council under Grant No. 162722, the Australian Institute for Nuclear Science and Engineering under grant number 884 and Insight Oceania Pty Ltd., Australia

M.L.F. Lerch (email mlerch@uow.edu.au), R. Ward, P.E. Simmonds A.B. Rosenfeld are with the Centre for Medical Radiation Physics, University of Wollongong, Wollongong, NSW, 2522, Australia, Ph. +61 242214954.

V.L.Pervertailo is with SPA-BIT, Kiev, Ukraine

S.R. Meikle is with the PET and Nuclear Medicine Department, Royal Prince Alfred Hospital, Sydney, NSW, Australia

G.N. Taylor is with the High Energy Physics Department, University of Melbourne, Melbourne, Vic, Australia
Significant research has been undertaken in the development of read out schemes of scintillator crystal configurations in an attempt to improve the spatial resolution of PET scanners $[1,2]$. We are developing a new Positron Emission Tomography (PET) detection module with depth of interaction capability. Pixilated scintillation crystals read out by silicon $8 \times 8$ photodiode arrays form the basis of the new module. The new photodiode arrays were designed by the Centre for Medical Radiation Physics and produced by SPA "Detector", Ukraine.

Silicon photodiodes represent the relatively cheap and robust end of the silicon based photodetector (PD) market. Improvement in the photodiode quantum efficiency at wavelengths around $420 \mathrm{~nm}$ (the peak emission wavelength of LSO), whist maintaining good photogenerated charge collection times, represents one of the biggest challenges in the photodiode design. We have designed a silicon PD to improve the PD quantum efficiency near the emission wavelength of LSO.

This article concentrates on comparison of the nuclear spectroscopic performance of the new PDs. In particular we are interested in measuring any quantitative improvement of the scintillation light collection efficiency of the diodes to see if it correlates with the measured improvement in the quantum efficiency.

\section{Detectors AND Methods}

\section{A. Detectors}

The photodiodes are of a planar design consisting of a $3 \times 3 \mathrm{~mm}^{2}$ active area and are $300 \mu \mathrm{m}$ thick. The detectors were produced by SPA-BIT "Detector", Ukraine on high resistivity nSi (Resistivity $5 \mathrm{k} \Omega \mathrm{cm}$ ). Three versions of the diode were produced: SPAD1, SPAD2 and SPAD3. SPAD 2 and 3 are both structurally similar to SPAD1. However, SPAD2 includes $p+$ strips at the surface of the p- layer aimed at enhancing the charge collection time as shown in Fig. 1. SPAD3 includes an additional antireflective layer to improve photon detection near the $430 \mathrm{~nm}$ (peak emission wavelength of the LYSO scintillator)[3]. 
The LYSO scintillator crystals have an emission spectrum that nominally peaks at $430 \mathrm{~nm}$ [4]. The absorption length of light with a wavelength of $430 \mathrm{~nm}$ is $0.1-0.2 \mu \mathrm{m}$ in silicon. In order to improve the sensitivity of the new photodiodes at wavelengths in the region of $400 \mathrm{~nm}$ we are using an (boron) ion implantation technique to produce the $\mathrm{p}^{-}$-layer that is optically coupled to the scintillator crystal. This technique allows the resulting electric field to extend very close to the $\mathrm{p}^{-}$ active surface, which has the dimensions of $3 \times 3 \mathrm{~mm}^{2}$ for each pixel element in our array, when the PD is under bias while still maintaining the potential across the $\mathrm{p}^{-}$surface. In effect we are able to improve the sensitivity of the PD in the blue spectral region, by reducing the effect of Auger recombination, while maintaining good charge collection properties to ensure fast timing signals for coincident detection techniques.
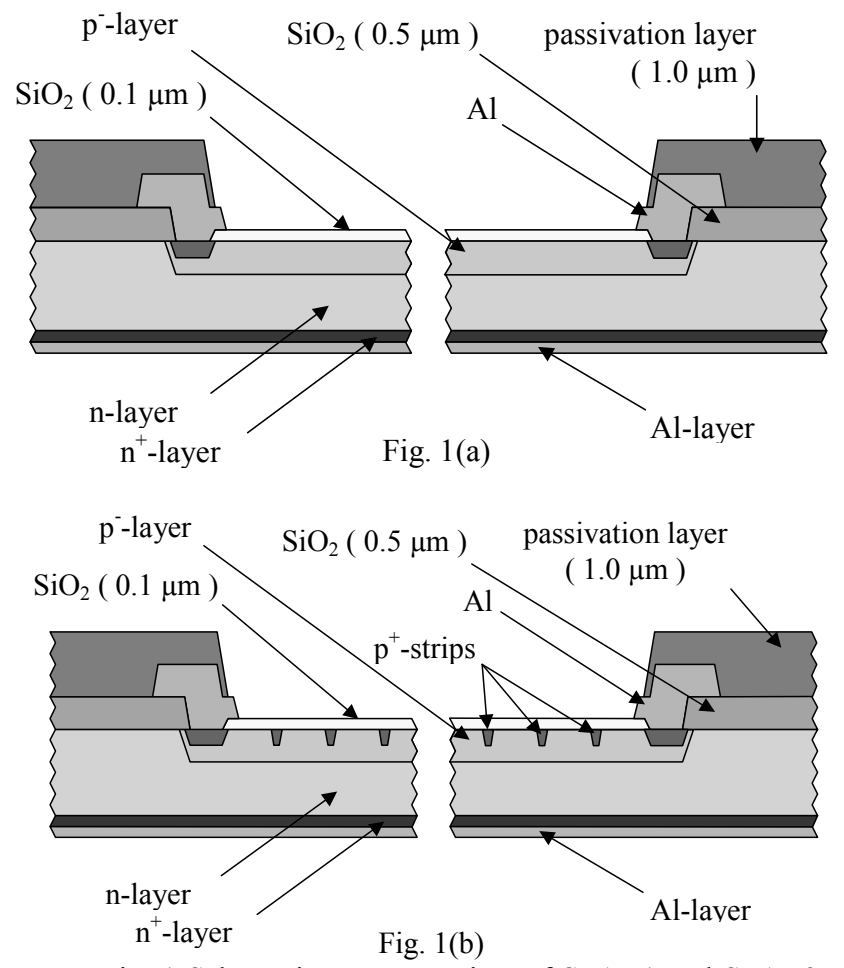

Fig. 1 Schematic cut-away view of SPAD1 and SPAD2. The two designs differ in that one has a uniform $\mathrm{p}^{-}$layer at the surface (Fig. 1a) and the other has $\mathrm{p}^{+}$strips embedded in the $\mathrm{p}^{-}$layer (Fig. 1b).

\section{B. Scintillators}

The LYSO scintillator material was purchased from Crystal Photonics Inc.(CPI), Sanford, Florida, USA. The material was subsequently cut into $3 \times 3 \times 3 \mathrm{~mm}^{3}$ cubes and polished and the absolute light output determined by A\&D Precision Co., Newton, MA, USA. The absolute light output was deduced to be $\sim 26$ photons $/ \mathrm{keV}$, which is $65 \%$ of that of $\mathrm{NaI}$ ( 40 photons $/ \mathrm{keV})$.

\section{Spectroscopy Experiments}

For gamma and X-ray spectroscopy experiments the spectroscopy readout arrangement comprised of an AMPTEK A250 preamplifier and a Canberra 2021, spectroscopy amplifier. A PC, using software supplied with the MCA, collected the data via an AMPTEK $^{\mathrm{TM}}$ 8000A MCA.

\section{Scintillator Light Collection Efficiency}

The complicated nature of the optical transparent layers forming the entrance window of the PD leads to a complicated quantum efficiency $(\mathrm{QE})$ curve as a function of incident optical photon angle (measured from the normal to the PD surface) [3]. The QE also varies with wavelength [3]. In order to compare one the gamma ray spectroscopy performance indicators of the different PD designs we have the "SCLICE". SLICE is the scintillator light collection efficiency and is simply the deduced number of photons detected by the PD divided by the estimated absolute number of optical photons emitted within the scintillator per gamma ray event $(\sim 13,300$ photons per $511 \mathrm{keV}$ event for our LYSO). We have assumed here that each optical photon detected produces one electron hole pair in the PD. For SLICE deductions direct X-ray spectroscopy (no scintillator crystal attached) data is used and was obtained under identical experimental conditions.

\section{RESULTS AND DISCUSSION}

\section{A. Quantum Efficiency}

Fig. 2 shows the external quantum efficiency (QE) of the SPAD3 relative to SPAD1 over the wavelength range of $380 \mathrm{~nm}$ to $600 \mathrm{~nm}$. The experimental method used to obtain this data is outlined in ref. [5]. The quantum efficiency of the SPAD3 is also shown on the same graph. The absolute responses were measured carefully under the same experimental conditions.

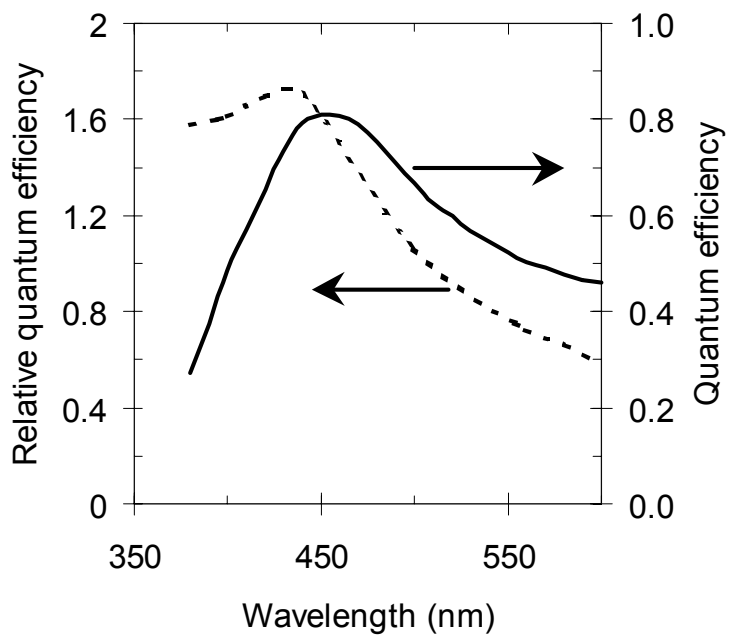

Figure 2. Relative quantum efficiency (QE) of SPAD3 to SPAD1 together with the external QE of SPAD3. 
The data indicates that the measured relative response and deduced quantum of SPAD3 is significantly enhanced compared to SPAD1 over the blue/violet spectral region. The enhancement in the blue/violet region reaches a maximum of $1.8 \mathrm{x}$ at $450 \mathrm{~nm}$. The internal quantum efficiency of these diodes has also been measured by us and is the same [3].

The deduced quantum efficiency for SPAD3 shown in Fig. 2 peaks at $450 \mathrm{~nm}$ where it is $81 \%$. Across several photodiodes the peak wavelength varied by $\pm 15 \mathrm{~nm}$ and the peak quantum efficiency varied by $\pm 5 \%$. This result is quite significant in that it indicates that SPAD3 is performing to its design specifications and hence is well suited for use with the LYSO scintillator whose emission wavelength peaks in the blue/violet spectral region.

\section{B. Gamma Ray Spectroscopy}

In gamma ray spectroscopy experiments the SPAD PD was optically coupled to a $3 \times 3 \times 3 \mathrm{~mm}^{3}$ LYSO crystal. The crystal has the same dimensions as that to be used in our detector module that incorporates pixilated crystals.

The best-measured energy resolution was $13.1 \%$ FWHM for the $511 \mathrm{keV}$ gamma rays and 11.2\% FWHM for the $662 \mathrm{keV}$ gamma rays from the ${ }^{22} \mathrm{Na}$ and ${ }^{137} \mathrm{Cs}$ source respectively. This result is shown in Fig 3 and was achieved using the SPAD3 PD, biased at $50 \mathrm{~V}$, to readout the painted LYSO scintillator, with a shaping time of $0.5 \mu \mathrm{s}$. Also shown in Fig 3 is the contribution to the FWHM from the readout electronics and PD system. The measured $8.2 \%$ is equivalent to $\sim 200 \mathrm{e}^{-} \mathrm{rms}$.

A variation in the energy resolution of $\pm 1 \%$ was observed by reading out the same LYSO crystal with several copies of the SPAD3 PD made from different parts of the wafer also used to make the silicon $8 \times 8$ arrays in the PET module.

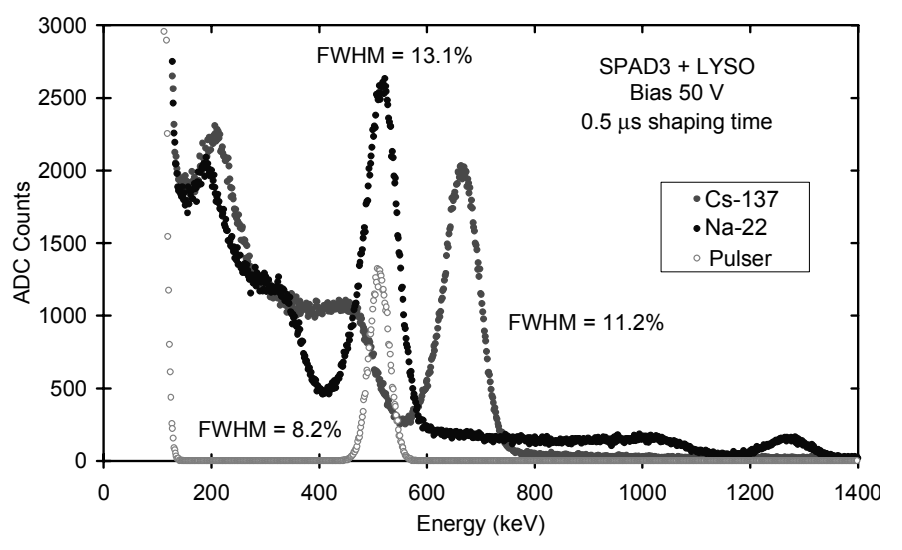

Figure 3. Pulse height spectrum of ${ }^{22} \mathrm{Na}$ and ${ }^{137} \mathrm{Cs}$.
Table 1 shows the experimentally measured energy resolution $(\mathrm{T}=298 \mathrm{~K})$ as a function of shaping time using the SPAD 3 photodetector at $50 \mathrm{~V}$ bias to read out the $3 \times 3 \times 3 \mathrm{~mm}^{3}$ painted LYSO scintillator crystal excited by a ${ }^{22} \mathrm{Na}$ source. The excellent energy resolution measured at short shaping times indicates that the PD can operate well under high count rate conditions if necessary, which is important in PET.

\begin{tabular}{|c|c|}
\hline Shaping Time $(\mu \mathrm{s})$ & $\begin{array}{c}\Delta \mathrm{E} / \mathrm{E}(\mathrm{SPAD} 3+\mathrm{LYSO}) \\
511 \mathrm{keV} \text { photopeak }\end{array}$ \\
\hline 0.10 & $17.8 \%$ \\
\hline 0.25 & $13.4 \%$ \\
\hline 0.50 & $13.1 \%$ \\
\hline 1.00 & $15.0 \%$ \\
\hline
\end{tabular}

Table 1 Energy resolution as a function of shaping time.

Fig. 4 shows the room temperature (298 K) pulse height spectrum when the LYSO crystal is excited by a ${ }^{22} \mathrm{Na}$ and ${ }^{137} \mathrm{Cs}$ source and read out by SPAD1 (top), SPAD2 (middle) and SPAD3 (bottom) PD respectively. In these experiments a $1.0 \mu \mathrm{s}$ shaping time was used. Fig. 4 shows the ADC channel on the Xaxis, which is proportional to the signal pulse from each detector. In this case the qualitative differences between each readout method is clearly observable. A quantitative comparison of the readout methods related to each PD is given in Table 2.

\section{Scintillator Light Collection Efficiency}

Fig. 4 shows the ADC channel on the $\mathrm{X}$-axis and energy on the $\mathrm{X} 2$-axis. In this case the relative improvement in the scintillator light collection efficiency (SLICE) for each version of the SPAD PD is clear as all experiments were performed under the same conditions. A quantitative estimate of the number of e-h pairs created by the optical scintillator photons detected by the PD can be obtained using a direct X-ray spectrum measured under the same operating and measuring conditions as shown in Fig. 5.

The $511 \mathrm{keV}$ photopeak occurs at the same channel as a 24.8 $\mathrm{keV}$ X-ray event directly detected in the PD. Therefore effectively $6850 \pm 70$ optical photons are detected by the PD per $511 \mathrm{keV}$ event in the LYSO crystal. Given that for our LYSO we expect $13,300 \pm 650$ photons per $511 \mathrm{keV}$ event the SLICE in this case is $53 \pm 0.4 \%$. A summary of the SLICE for each PD is shown in Table 2.

Table 2 shows that an $18 \%$ improvement is observed in the scintillation light collection efficiency between the SPAD1 and SPAD3 readout scheme. This improvement is very significant in the application of these PDs for PET but is far less than the increase in measured external quantum efficiency. In order to understand this observation one must take into account the angular dependence and wavelength dependence of the optical response in combination with the angular distribution of the 
optical photons incident on the PD surface. We are doing this work as part of a separate study [3].
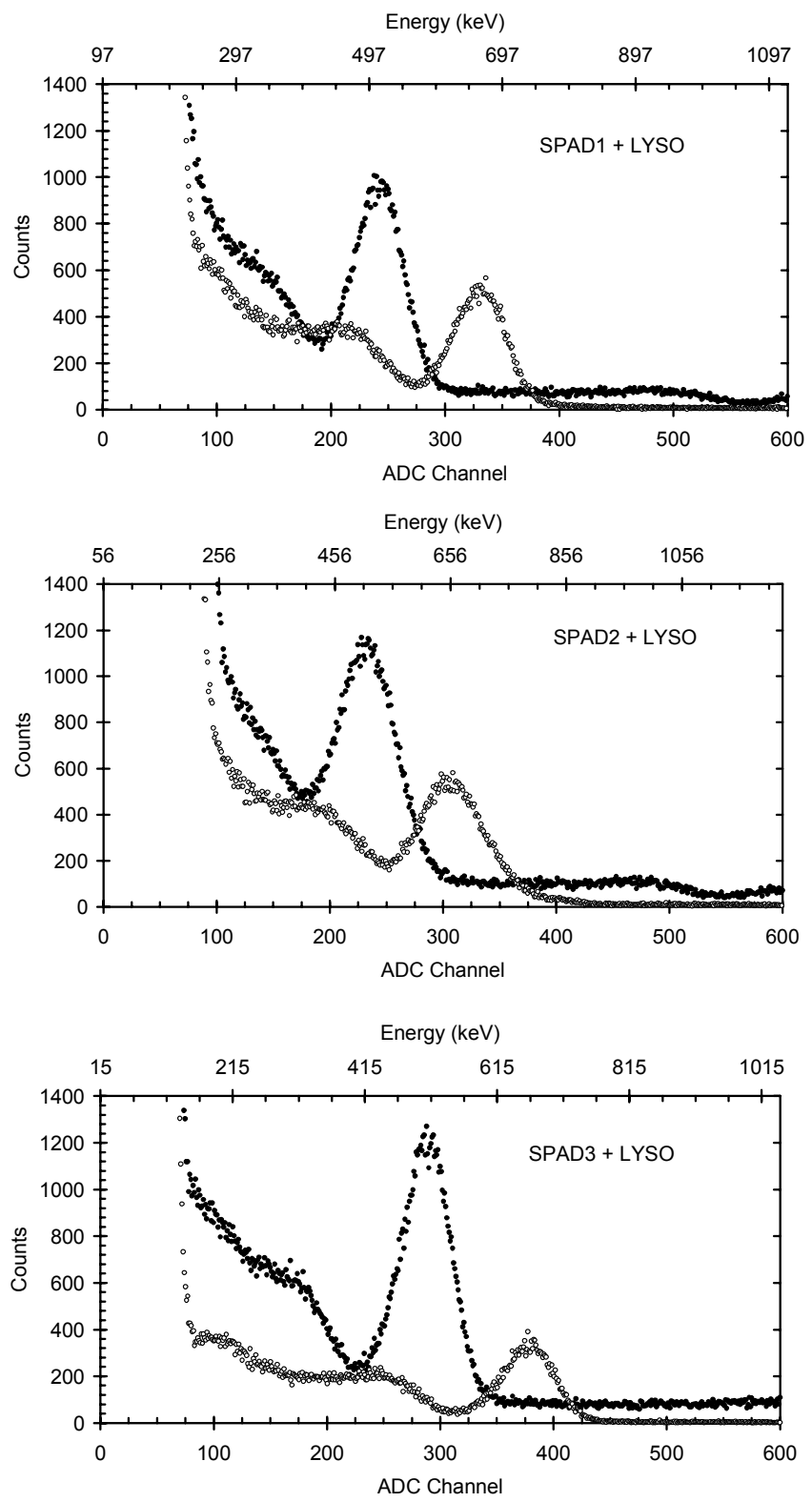

Fig. 4 Room temperature $(298 \mathrm{~K})$ pulse height spectrum when the LYSO crystal is excited by a ${ }^{22} \mathrm{Na}$ and ${ }^{137} \mathrm{Cs}$ source and read out by SPAD1 (top), SPAD2 (middle) and SPAD3 (bottom) PD respectively.

The small decrease of $3 \pm 0.3 \%$ in the scintillation light collection efficiency between the SPAD1 and SPAD2 readout scheme was less significant than expected. The reduction is due to the addition of $\mathrm{p}+$ strips within the active layer of the SPAD2 $\mathrm{PD}$ effectively reducing the internal quantum efficiency of the PD. The $p+$ strips occupy approximately one third of the active area. The minor reduction in efficiency could be tolerated if a significant improvement in the timing properties of the PD is observed. This study is currently on going.

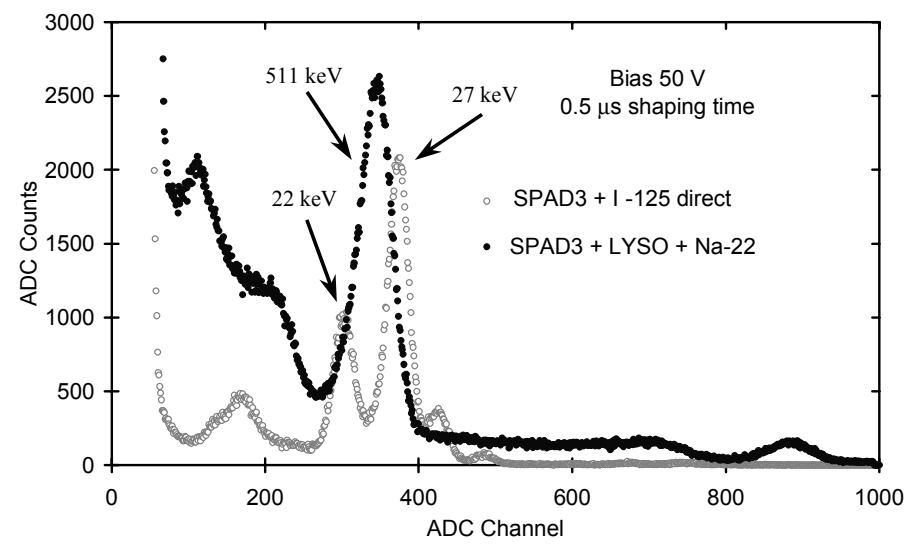

Fig. 5 Room temperature pulse height spectrum of an LYSO crystal excited by a ${ }^{22} \mathrm{Na}$ source and read out by SPAD3 overlayed with an ${ }^{125} \mathrm{I}$ spectrum directly detected in the same PD. All operating and measurement conditions are identical.

\begin{tabular}{|c|c|l|}
\hline PD & $\begin{array}{c}\Delta E / E(L Y S O) \\
511 \mathrm{keV} \text { peak }\end{array}$ & $\begin{array}{l}\text { Scintillator Light Collection } \\
\text { Efficiency (SLICE) }\end{array}$ \\
\hline SPAD1 & $15.1 \%$ & $\begin{array}{l}5800 \text { optical photons detected or } \\
43.6 \pm 0.4 \%\end{array}$ \\
\hline SPAD2 & $16.8 \%$ & $\begin{array}{l}5800 \text { optical photons detected or } \\
42.1 \pm 0.4 \%\end{array}$ \\
\hline SPAD3 & $13.1 \%$ & $\begin{array}{l}5800 \text { optical photons detected or } \\
53.0 \pm 0.4 \%\end{array}$ \\
\hline
\end{tabular}

Table 2 Comparison of the measured energy resolution (shaping time of $0.50 \mu \mathrm{s}$ ) and scintillator light collection efficiency (SLICE) for SPAD 1, SPAD2 and SPAD3.

\section{CONCLUSION}

We have characterised the custom designed silicon photodiodes using nuclear spectroscopic techniques that will be used in the development of a new detector module for positron emission tomography. The SPAD3 PDs have a measured quantum efficiency of $81 \%$ at $450 \mathrm{~nm}$. We have measured an energy resolution of $13.1 \%$ FWHM for the $511 \mathrm{keV}$ gamma rays from a ${ }^{22} \mathrm{Na}$ source. The new photodiodes are well suited for the 
readout of scintillators whose emission wavelength peak in the blue/violet spectral region.

\section{REFERENCES}

[1] Pichler, B.J., Bernecker, F., Boning, G., Rafecas, M., Pimpl, W., Schwaiger, M., Lorenz, E., Ziegler, S.I., "A 4x8 APD array, consisting of two monolithic silicon wafers, coupled to a 32channel LSO matrix for high-resolution PET". IEEE Trans. Nucl. Sci., Vol. 48 (4), $1391-1396,2001$

[2] Levin, C.S., "Design of a high resolution and high sensitivity scintillation crystal array with nearly perfect light collection" Presented at 2001 IEEE NSS and MIC Conference, San Diego, USA

[3] R.D. Ward, M.L.F. Lerch, P.E. Simmonds, G. Takacs, V. Perevertailo, and A.B. Rosenfeld, "Angular dependence of response in a New Silicon Photodiode for use in PET" presenting at IEEE NSS/MIC conference 2003.

[4] Saoudi, A., Pepin, C., Pepin, C., Houde, D., and Lecomte, R., "Scintillation light emission studies of LSO scintillators", IEEE Trans. Nucl. Sci., Vol. 46 (6), 1925 -1928, 1999

[5] M.L.F.Lerch, A.B.Rosenfeld, P.E. Simmonds, G.N.Taylor, S.R.Meikle, V.L. Perevertailo "Spectral Characterization of blueenhanced silicon photodiode", IEEE Trans. on Nucl. Sci., NS-48, N-4, 1220-1224, 2001 